\title{
Cell cycle-dependent resolution of DNA double-strand breaks
}

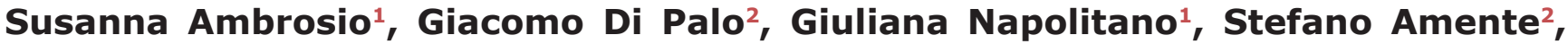 \\ Gaetano Ivan Dellino ${ }^{3,4}$, Mario Faretta ${ }^{3}$, Pier Giuseppe Pelicci ${ }^{3,4}$, Luigi Lania ${ }^{2}$, \\ Barbara Majello ${ }^{1}$ \\ ${ }^{1}$ Department of Biology, University of Naples 'Federico II', Naples, Italy \\ ${ }^{2}$ Department of Molecular Medicine and Medical Biotechnologies, University of Naples 'Federico II', Naples, Italy \\ ${ }^{3}$ Department of Experimental Oncology, European Institute of Oncology, Milan, Italy \\ ${ }^{4}$ Department of Oncology and Haemato-oncology, University of Milan, Italy \\ Correspondence to: Luigi Lania, e-mail: Iania@unina.it \\ Barbara Majello, e-mail: majello@unina.it \\ Keywords: cell-cycle, DSB repair, site-specific DSBs, AsiSl restriction enzyme \\ Received: November 05, $2015 \quad$ Accepted: November 27, $2015 \quad$ Published: December 17, 2015
}

\section{ABSTRACT}

DNA double strand breaks (DSBs) elicit prompt activation of DNA damage response (DDR), which arrests cell-cycle either in $G_{1} / S$ or $G_{2} / M$ in order to avoid entering $S$ and $M$ phase with damaged DNAs. Since mammalian tissues contain both proliferating and quiescent cells, there might be fundamental difference in DDR between proliferating and quiescent cells (or $\mathbf{G}_{0}$-arrested). To investigate these differences, we studied recruitment of DSB repair factors and resolution of DNA lesions induced at site-specific DSBs in asynchronously proliferating, $\mathbf{G}_{0}{ }^{-}$, or $\mathbf{G}_{\mathbf{1}^{-}}$ arrested cells. Strikingly, DSBs occurring in $G_{0}$ quiescent cells are not repaired and maintain a sustained activation of the p53-pathway. Conversely, re-entry into cell cycle of damaged $G_{0}$-arrested cells, occurs with a delayed clearance of DNA repair factors initially recruited to DSBs, indicating an inefficient repair when compared to DSBs induced in asynchronously proliferating or $G_{1}-$ synchronized cells. Moreover, we found that initial recognition of DSBs and assembly of DSB factors is largely similar in asynchronously proliferating, $\mathbf{G}_{0}-$, or $\mathbf{G}_{1}$-synchronized cells. Our study thereby demonstrates that repair and resolution of DSBs is strongly dependent on the cellcycle state.

\section{INTRODUCTION}

Eukaryotic genome is constantly being challenged by various endogenous and exogenous insults. These damaging events include crosslinks, base modifications, base mismatches, stalled replication forks, single-strand breaks (SSBs), and particularly dangerous double-strand breaks (DSBs). To deal with such dangerous insults, eukaryotes possess an array of distinct pathways to monitor and repair damaged DNA.

The initial phases of DSB recognition and recruitment of repair factors are now quite elucidated. Following DSB the MRE11/RAD50/NBS1 complex senses a DSB within seconds and then activates PI3K-like kinases ATM (ataxia-telangiectasia mutated) protein kinase, a large Ser/Thr kinase of the PI3K- like kinase family, which also includes DNA-PKcs (DNA-dependent protein kinase catalytic subunit), and ATR [1-4]. ATM then phosphorylates histone H2AX on Ser139 (named $\gamma \mathrm{H} 2 \mathrm{AX}$ when phosphorylated) in DSB adjacent chromatin. The primary function of $\gamma \mathrm{H} 2 \mathrm{AX}$ is to recruit its decoder, MDC1 (mediator of DNA damage checkpoint protein 1), which recognizes the phosphorylated Ser139 epitope on $\gamma \mathrm{H} 2 \mathrm{AX}$. $\gamma \mathrm{H} 2 \mathrm{AX}$ bound MDC1 recruits in turn more MRN complexes (via an interaction with NBS1) and thus initiates a positive ATM feedback loop that leads to the amplification of the $\gamma \mathrm{H} 2 \mathrm{~A}$.X chromatin domain [5-8]. Concomitant with the assembly of DNA repair factors at DNA lesion, DSB response activates DNA-damage checkpoints (DDR), and diffusible signaling events that can arrest cell cycle progression either in $G_{1}$ or $G_{2}$ to allow for DNA repair 
and prevent transmission of damaged DNA to daughter cells $(9,10)$. However, it must be emphasized that the tissue and organs of mammals consist of different cell types, including dividing, non-dividing and stem cells that coexist in several tissues, in separate yet adjoining locations [11]. Normal mammalian cells possess unique regulatory mechanisms to shift from a quiescent state to a proliferative state and dysregulation of these mechanisms might result in malignant transformation. Cellular quiescence and the capacity to enter the proliferation cycle are critical for maintaining tissue homeostasis $[12,13]$.

During interphase, DSB can be repaired in a cellcycle dependent manner by two major mechanisms: classical non-homologous end joining (NHEJ, during $\mathrm{G}_{1}$ phase) or homologous recombination repair (HR, mainly in $\mathrm{S}-\mathrm{G}_{2}$ phases) [14-16]. Several studies unveiled cell cycle-regulated circuits that govern DSB repair pathway choice to ensure that NHEJ dominates in $G_{1}$ and $H R$ is favored from $S$ phase onward [17-21]. Although cell-cycle phase contributes to this choice, these pathways coexist in $\mathrm{S}$ - and $\mathrm{G}_{2}$-phases, thus implying that other factors participate in this decision such as chromatin context in which DSB occurs [22-24].

It is now well established that DDR differs in mitotic and interphase cells $[25,26]$. It has been shown that DDR is dampened during mitosis. Cells have evolved mechanisms to suppress DSB repair during $M$ phase to prevent genome instability [27, 28]. Clearly, different molecular mechanisms involved in DNA repair occurring at specific cell cycle phases have been evolved, and recruitment of DSB repair factors and resolution of DNA lesions induced at site-specific DSBs occurring during different phases of the cell cycle could be instrumental to investigate these differences.

To avoid potential anomalies associated with transformed cell lines, we produced a cellular system suitable to the induction of specific DSBs in the immortalized non-tumorigenic epithelial cell line MCF10A. We stable transfected these cells with a welldocumented AsISI-inducible vector that express the 8-base restriction endonuclease AsiSI fused to a modified oestrogen receptor ligand binding domain that induces nuclear localization of the enzyme after administration with 4hydroxytamoxifen (4OHT) causing the rapid and reproducible induction of about 150 sequence-specific DSBs across the genome [23, 29-31]. This system (MCF10A-AsiSI-ER) offers the opportunity to study the wave of repair events occurring at defined stages of the cell cycle in a defined and reproducible manner.

We found the DSBs occurring in $\mathrm{G}_{0}$ quiescent cells are irreparable with a sustained activation of the p53pathway. Conversely, re-entry into cell cycle of damaged $\mathrm{G}_{0}$-arrested cells shows a delayed clearance of recruited DNA repair factors bound at DSBs, indicating inefficient repair. This study thereby demonstrates the crucial role of cell cycle phases in repair and resolution of DSBs.

\section{RESULTS}

\section{Induction of specific DSBs in non-tumorigenic epithelial MCF10A cells}

To investigate DSB damage and avoid potential anomalies associated with transformed cell lines, we sought to produce a cellular system suitable to the induction of specific DSBs in the immortalized nontumorigenic epithelial cell line MCF10A. To this end MCF10A cells were transduced with a retroviral vector expressing the fusion protein between the HA-tagged AsiSI restriction enzyme and a modified hormone-binding domain from the estrogen receptor. Following drug selection, one cell clone was isolated (named MCF10-AsiSIER) and the effects of 4OHT administration at different time points were analyzed by indirect immunofluorescence. Exposure to 4OHT for 2 hours resulted in nuclear accumulation of the AsiSI fusion protein, as detected by anti-HA-tag antibodies (Figure 1A). This was accompanied by a significant increase in the number of DNA damaged foci, visualized with antibodies against S139-phosphorylated histone $\gamma \mathrm{H} 2 \mathrm{AX}$ (Figure 1B). 4OHT was removed from the medium after 2 hours and cells cultivated for additional 4 and 8 hours (Recovery). As shown in Figure 1A, the nuclear localization of HA-AsiSIER was strongly reduced after 4 hours of recovery, and barely detectable after 8 hours, indicating that the HA-AsiSI-ER endonuclease was not active anymore (Figure 1A). The generation of the MCF10-AsiER clone enabled us to investigate recruitment of DNA damaging factors at specific DSBs by using ChIP-based approaches. As initial test we focused on the AsiSI sites on chromosomes 1 and 6 at which $\gamma \mathrm{H} 2 \mathrm{AX}$ recruitment had been observed and documented by treatment of U2OS AsiSI cells [29]. We conducted ChIP assays with antibodies against some of the DDR components and we used sequences of published primer sets (listed in Table S2). As illustrated in Figure 1C, we observed increased enrichment of $\gamma \mathrm{H} 2 \mathrm{AX}, \mathrm{NBS} 1$, and XRCC4 at the Chr1 and Chr6 AsiSI sites. These results confirm that recruitment of these factors in MCF10-AsiER paralleled the effects observed in previously described clonal population of U2OS-AsiER cells.

\section{DSBs induce DDR activation followed by efficient repair in MCF10A proliferating cells}

Following the generation of DSBs, DDR promotes cellular DNA-repair activities with a concomitant transient arrest of cell-cycle progression (checkpoint function) until DNA damage has been removed. To analyze the transient arrest of cell-cycle progression following induction of DSBs, proliferating MCF10-AsiSIER cells were treated for 2 hours with $4 \mathrm{OHT}$ and then allowed to recover in 
the absence of 4 OHT for 24, 48 and 72 hours. Samples were analyzed for cell-cycle distribution, DDR activation, and ChIP accumulation of $\gamma \mathrm{H} 2 \mathrm{AX}$ and NBS1 at specific AsiSI sites. Cell cycle analysis showed that AsiSIdependent DSBs induced a significant G2 arrest, which was completely resolved after $72 \mathrm{hr}$ of Recovery (Figure 2A). As shown in Figure 2B, p53-Ser15 phosphorylation increased after 4OHT treatment and its levels decreased 3 days after the removal of the DNA damage insult.

DDR cascade begins with the detection of DSBs by the MRN (MRE11-RAD50-NBS1) complex, which recruits and activates different PIKK kinases (ATM, ATR and DNA-PK), each capable to phosphorylate H2AX at Ser139 [3-5]. To analyze the efficiency of these steps detecting DSBs and to monitor the resolution of DNA damage-associated $\gamma \mathrm{H} 2 \mathrm{AX}$ and NBS1 accumulation at defined AsiSI sites we performed ChIP with anti- $\gamma \mathrm{H} 2 \mathrm{AX}$ and -NBS1 antibodies. Following the robust increase of $\gamma \mathrm{H} 2 \mathrm{AX}$ and NBS1 signals at the AsiSI sites after 4OHT treatment, we observed their progressive reduction within 24 hours (Figure 2C and Supplementary Figure 1).

Collectively, these data indicate that induction of DSBs in asynchronously proliferating MCF10 cells promotes a robust DDR activation, which is followed by an efficient wave of repair leading to a progressive reduction of DDR after DSBs onset.

\section{DSBs in quiescent MFC10 cells are irreparable and cause a sustained activation of the p53- pathway}

In mammalian tissues, cells are in both proliferating and quiescent states depending on the given tissue and these two different populations may also coexist in several tissues, in separate yet adjoining locations. However, comparative study of these two distinct cell cycle states regarding the capability to sense and resolve DNA DSB damaging insults has been poorly characterized. To address this issue and investigate if quiescent or proliferating cells equally sense and resolve DSBs over time, we took advantage of the MCF10AsIER cells which can be induced in a quiescent state by growth factors deprivation for 2 days (referred to as $G_{0}$ cells). $G_{0}$ cells were then treated or not with 4 OHT for 2 hours to induce DSBs. The efficiency of DSB induction at each AsiSI site was measured in these two conditions by ChIP-sequencing of proliferating and $\mathrm{G}_{0}$-arrested cells using the anti- $\gamma \mathrm{H} 2 \mathrm{AX}$ antibody. Similarly to ChIP data already available for U2OS cells [22], $\gamma \mathrm{H} 2 \mathrm{AX}$ showed a typical pattern with signals encompassing the DSBs for $1-2 \mathrm{Mb}$ around the AsiSI sites, with the typical signal drop occurring exactly at the restricted AsiSI sites (Figure 3, and Supplementary Figure 2).
A
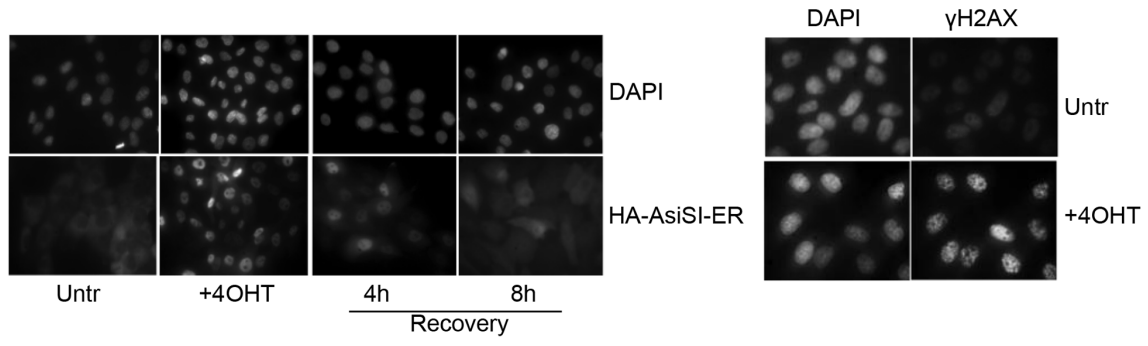

C
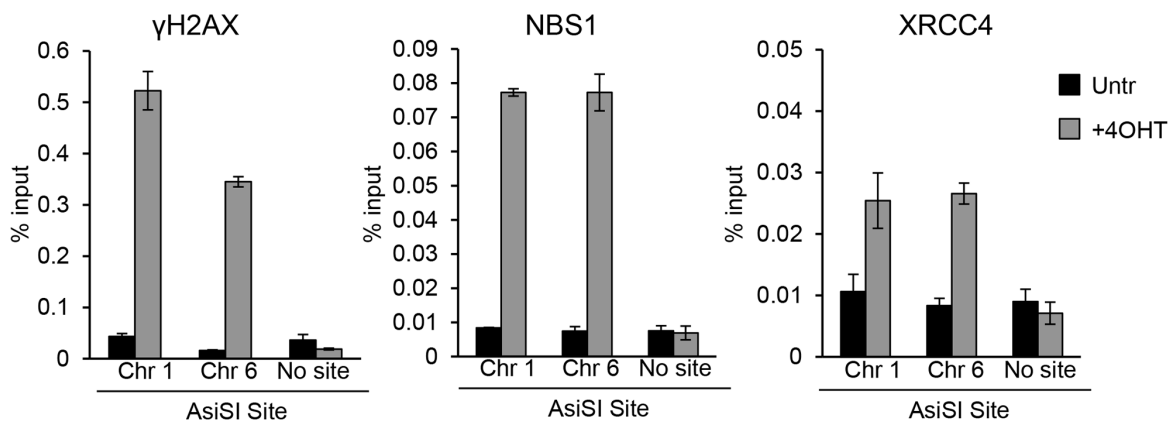

Figure 1: 40HT treatment triggers DSBs formation at AsiSI sites in MCF10A. A. MCF10A-AsiSI-ER cells were treated for $2 \mathrm{~h}$ with $4 \mathrm{OHT}$ or vehicle (Untr) and then released into fresh medium for 4 and $8 \mathrm{~h}$ (Recovery). Cells were fixed and processed for antiHA immunofluorescence and DAPI staining. B. MCF10A-AsiSI-ER cells were treated for $2 \mathrm{~h}$ with $4 \mathrm{OHT}$ and stained with anti- $\gamma \mathrm{H} 2 \mathrm{AX}$ antibody. DAPI staining of nuclei is shown. C. MCF10A-AsiSI-ER cells were treated as above and ChIP experiments were performed using antibodies against $\gamma \mathrm{H} 2 \mathrm{AX}$, NBS1 and XRCC4. Real-time qPCR was done on ChIP materials using primers listed in Supplementary Table 2. Amplicon far from any AsiSI site was analyzed as negative control. Data are from independent experiments with $\mathrm{SD}(n=3)$. 
Most importantly, we confirmed the results by analyzing $150 \gamma \mathrm{H} 2 \mathrm{AX}$ peaks and found that $\gamma \mathrm{H} 2 \mathrm{AX}$ mapped with similar efficiency in both $\mathrm{G}_{0}$ and proliferating cells (Figure 3 and Supplementary Figure 2 and Table 3). From these observations we assessed that the efficiency of DSBs induced in either proliferating or $\mathrm{G}_{0}$-arrested cells is largely similar. Next, we followed $\gamma \mathrm{H} 2 \mathrm{AX}$ and 53BP1 foci formation by immunofluorescence in damaged $\mathrm{G}_{0}$-arrested cells. We found that, similarly to proliferating cells, $\mathrm{G}_{0}$-arrested cells exposed to $4 \mathrm{OHT}$ for 2 hours showed a drastic induction of $\gamma \mathrm{H} 2 \mathrm{AX}$ and 53BP1 foci. However, we found that DSB in $\mathrm{G}_{0}$ arrested cells were not repaired, with a persistent accumulation of $\gamma \mathrm{H} 2 \mathrm{AX}$ and 53BP1 foci up to 5 days after DSB induction, thus suggesting an impaired repair proficiency (Figure 4A). Accordingly, $\gamma \mathrm{H} 2 \mathrm{AX}$ ChIP data showed a sustained accumulation of $\gamma \mathrm{H} 2 \mathrm{AX}$ signal at the AsiSI sites (Figure 4B). We cannot exclude that DSBs might normally repaired in quiescent cells but fail to recover normal chromatin arrangement after repair. However, sustained expression of the P-p53-p21 axis was observed, suggesting that the DDR p53 pathway operates in $\mathrm{G}_{0}$-damaged cell. Interestingly, $\mathrm{p} 21$, which was present at high levels in quiescent cells, was further up-regulated after damage (Figure 4C). Moreover we found that damaged $\mathrm{G}_{0}$-arrested cells underwent to apoptosis after 3 days of OHT treatment, as documented by a robust increase of the cleaved PARP1 protein in damaged cells. PARP1 cleavage was not observed in vehicle treated undamaged cells (Veh) that could be kept in culture up to 10 days (Figure 4D and data not shown).

Next we investigated whether the lack of DNA repair efficiency was a consequence of different expression levels of DDR genes in $\mathrm{G}_{0}$-arrested cells compared to proliferating cells. We comparatively quantified expression levels of DDR genes in $\mathrm{G}_{0}$-arrested versus asynchronous proliferating cells by qRT-PCR and Western blot analysis. As shown in Figure $4 \mathrm{E}$ and $4 \mathrm{~F}, \mathrm{G}_{0}$-arrested cells expressed the analyzed DDR factors at comparable levels with proliferating cells except for RAD51 and ATR. The low expression levels of RAD51 and ATR are consistent with their role in $G_{1} / S$ phases. Data obtained showed that no significant differences were seen between $\mathrm{G}_{1} / \mathrm{S}$ and asynchronous proliferating cells.

B

A
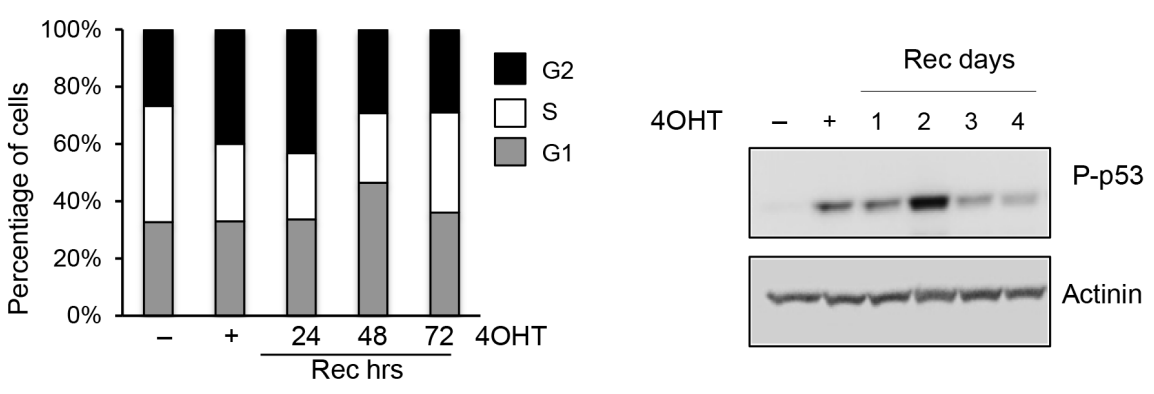

C

$\mathrm{YH} 2 \mathrm{AX}$

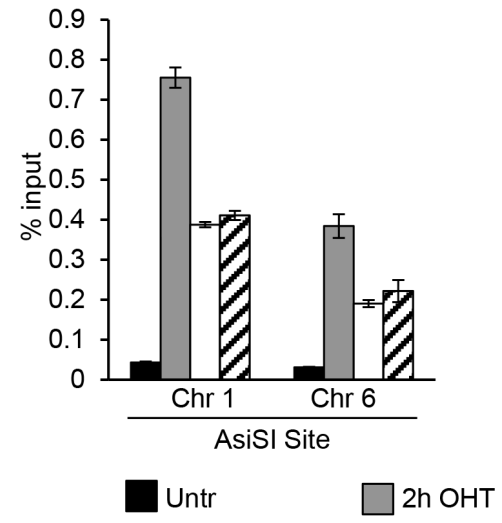

NBS1

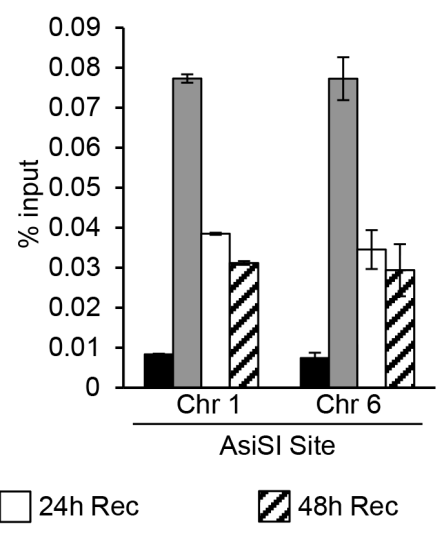

Figure 2: AsiSI-induced DSBs trigger DDR activation followed by efficient wave of repair. A. Cell cycle distribution of asynchronously growing MCF10A-AsiSI-ER treated for $2 \mathrm{~h}$ with $4 \mathrm{OHT}$ then released into fresh medium and collected as indicated. DNA content of propidium iodide stained cells was determined by flow cytofluorimetry. B. Total cell extracts from proliferating MCF10A-AsiSI before and at the indicated times after 4OHT removal were probed with anti-phospho-p53 and normalized for actinin. C. ChIP against $\gamma \mathrm{H} 2 \mathrm{AX}$ and NBS1 in MCF10A-AsiSI-ER treated for $2 \mathrm{~h}$ with $4 \mathrm{OHT}$ then released into fresh medium, collected as indicated and analyzed by qPCR. Data are from independent experiments with $\mathrm{SD}(n=3)$. 
From these findings we concluded that $\mathrm{G}_{0}$-arrested cells lack DNA repair proficiency, but retain the capability to activate DNA damage response.

\section{Cell-cycle reentry induces a delayed resolution of DSBs}

Our findings demonstrated that DSB occurring in $\mathrm{G}_{0}$-arrested cells are not repaired. We then sought to determine whether reentry of $\mathrm{G}_{0}$-damaged cells in cell cycle progression might recover DNA repair proficiency. First, we monitored the cell cycle re-entry of MCF10AsiER cells upon 2 days of starvation. Cells were grown in minimal medium for 2 days and then cell cycle re-entry was induced by addition of medium containing growth factors (hydrocortisone, EGF, insulin, cholera toxin). Flow cytometry analysis revealed an increase of $\mathrm{S}$ phase cells 8 hours after growth factors addition, with a concomitant increase of Ki67 levels compared to starved cells; moreover after $24 \mathrm{~h}$, percentages of cell cycle phases

A

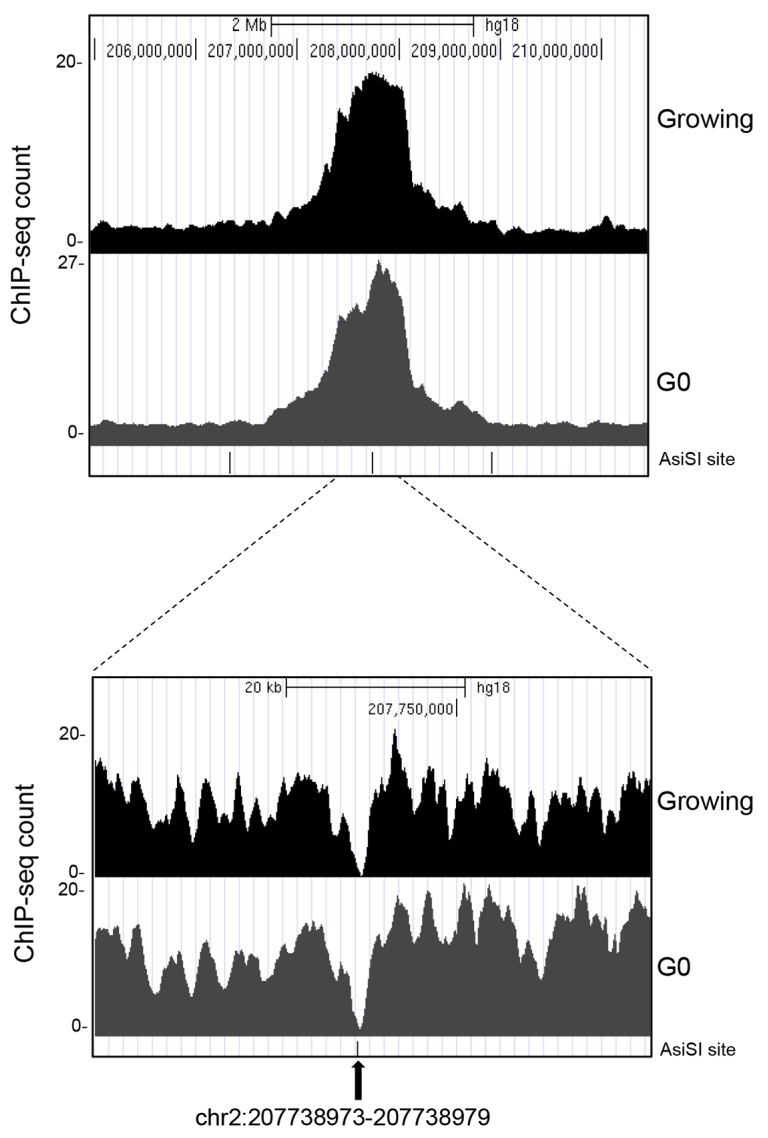

B

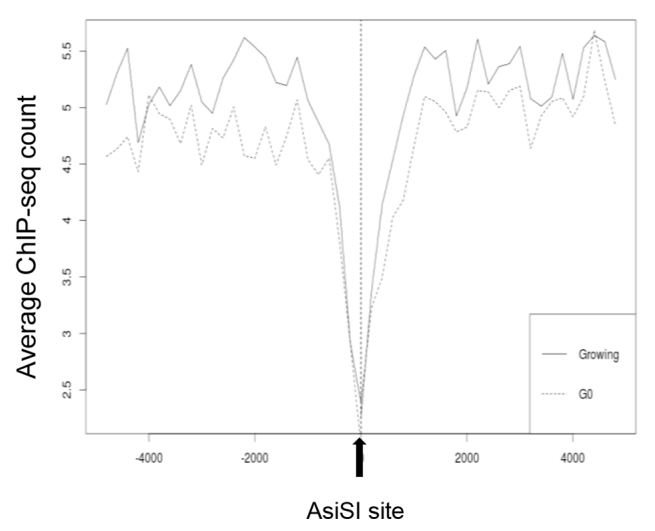

Figure 3: ChIP-seq analyses in proliferating and $\mathrm{G}_{0}$-arrested MCF10A-AsiSI-ER cells after 4OHT treatment (2 h), using anti- $\gamma \mathbf{H} 2 \mathrm{AX}$ antibody. Panel A. show the profiles of $\gamma \mathrm{H} 2 \mathrm{AX}$ around a selected AsiSI site in both proliferating and $\mathrm{G}_{0}$-arrested cells. B. Averaged $\gamma \mathrm{H} 2 \mathrm{AX}$ signals of proliferating and $\mathrm{G}_{0}$ cells over a 10-kb windows and centered at the AsiSI site. 
and Ki67 levels were largely similar to growing control cells (Figure 5A and 5B).

Damaged $\mathrm{G}_{0}$-arrested cells showed an accumulation in $G_{2}$ phase with a delayed cell-cycle re-entry (Figure 5C) with abnormal accumulation on both $\mathrm{G}_{1}$ and $\mathrm{G}_{2}$ phases. As shown in Figure 5D, P-p53 levels increased after DNA damage and accumulation of P-p53-Ser15 was detected up to 2 days after DSBs followed by a sharp decline at 3 and 5 days after recovery, suggesting resolution of induced damage.
Finally we monitored accumulation of the DNA repair factors $\gamma \mathrm{H} 2 \mathrm{AX}$ and NBS1 at specific AsiSI sites at different time-points after cell-cycle reentry. ChIP data demonstrated that $\gamma \mathrm{H} 2 \mathrm{AX}$ and NBS1 factors were rapidly recruited to the AsiSI sites. However, compared to DNA damage in proliferating cells, in damaged $\mathrm{G}_{0}$ arrested cells we found a persistent accumulation of both $\gamma \mathrm{H} 2 \mathrm{AX}$ and NBS1 at DSBs (Figure 5E).

Collectively, these data clearly indicate that cell cycle re-entry of damaged $\mathrm{G}_{0}$-arrested cells induces

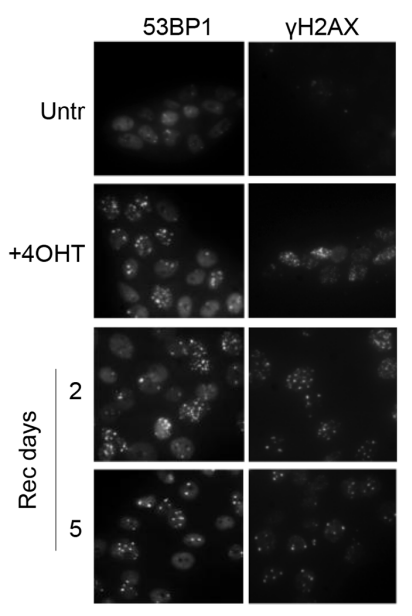

C

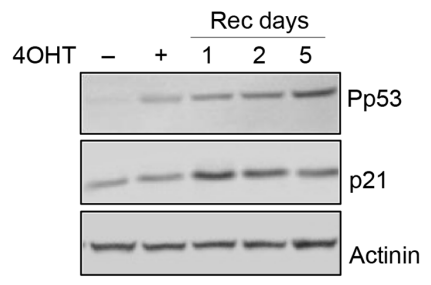

B

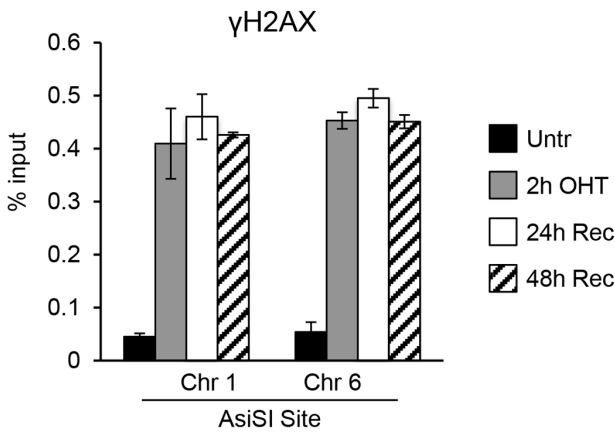

D

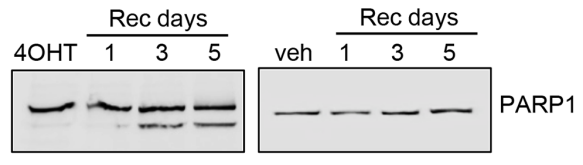

E

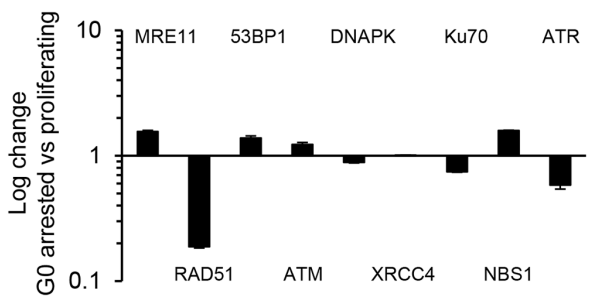

$\mathbf{F}$

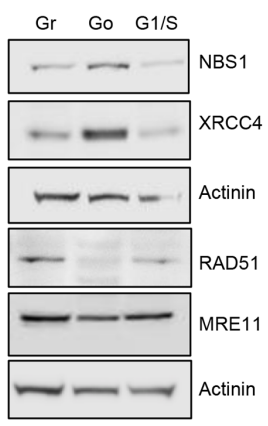

Figure 4: G $_{\mathbf{0}}$-arrested MCF10-AsiSIER cells lack DNA repair proficiency. A. MCF10A-AsiSIER cells were arrested in G phase through grow factors deprivation for $40 \mathrm{~h}$, treated with $4 \mathrm{OHT}$ for $2 \mathrm{~h}$ then kept in medium without grow factors, and analyzed at the indicated times after 4OHT removal by immunofluorescence with anti-53BP1 and anti- $\gamma \mathrm{H} 2 \mathrm{AX}$ antibodies, respectively. B. Recruitment of $\gamma \mathrm{H} 2 \mathrm{AX}$ at AsiSI sites (Chr. 1 and 6) was determined by ChIP assays. C. Western blotting was performed using phospho-p53 antibodies and p21. D. PARP1 detection of both full-length and cleaved protein fragments; western blotting of $\mathrm{G}_{0}$-arrested MCF10-AsiSI-ER treated with $4 \mathrm{OHT}$ or vehicle, collected at the indicated time points after $4 \mathrm{OHT}$ removal. E. DDR factors $\mathrm{mRNAs}$ expression analysis of $\mathrm{G}_{0}$-arrested MCF10-AsiSI-ER through quantitative RT-PCR. Expression profiles were normalized against proliferating cells. F. Western blot of protein extracts of Growing, $\mathrm{G}_{0}$ and G1/S MCF10-AsiSI-ER cells using the indicated antibodies. Actinin has been probed as loading control for different blots. 
delayed resolution of DSBs compared to proliferating damaged cells; following recruitment of repair factors, the progressive reduction in time of accumulation of these factors in $\mathrm{G}_{0}$-damaged cells was clearly delayed compared to what observed in asynchronously proliferating MCF 10 cells.

\section{Efficient resolution of DSB induced at $G_{1} / S$ phase}

Our findings demonstrated that cell cycle reentry of $\mathrm{G}_{0}$-damaged cells allows resolution of DSBs albeit with a delayed efficiency compared to proliferating cells, suggesting that transition to $G_{1} / S$ phases might be required for DSB resolution. We then sought to determine repair proficiency in synchronized $\mathrm{G}_{1} / \mathrm{S}$-damaged cells. As shown by FACS data, 8 hours after cell cycle re-entry, $\mathrm{G}_{0}$-arrested MCF10-AsIER cells were synchronized in late $\mathrm{G}_{1} / \mathrm{S}$ phase (Figure $5 \mathrm{~A}$ ). Synchronized cells were exposed for 2 hours to 4OHT treatment and then allowed to recover: cell samples were collected at different times after the 4OHT pulse and analyzed for p53 activation and accumulation of DSB factors at specific AsiSI sites. $\mathrm{G}_{1}$-damaged cells exhibited a robust p53 activation (Figure 6A), and accumulation of the DNA repair factors $(\gamma \mathrm{H} 2 \mathrm{AX}$ and NBS1) at specific AsiSI sites at different time-points following DNA damage. ChIP data showed
A

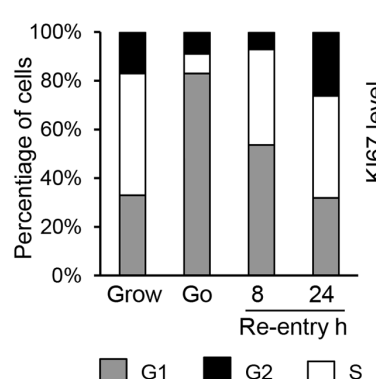

B

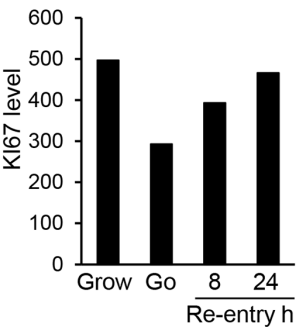

C

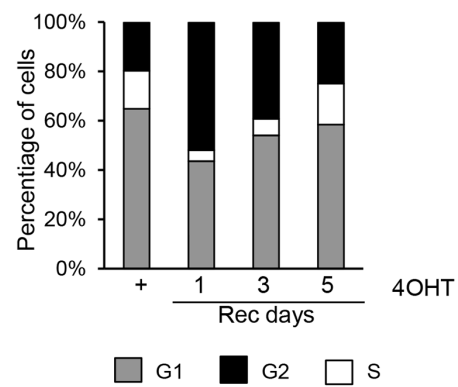

D

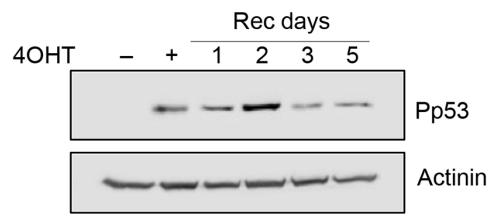

E

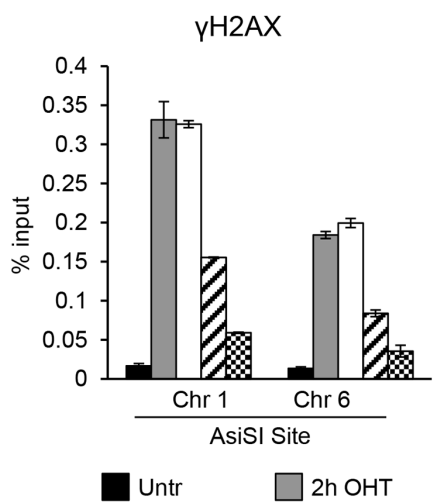

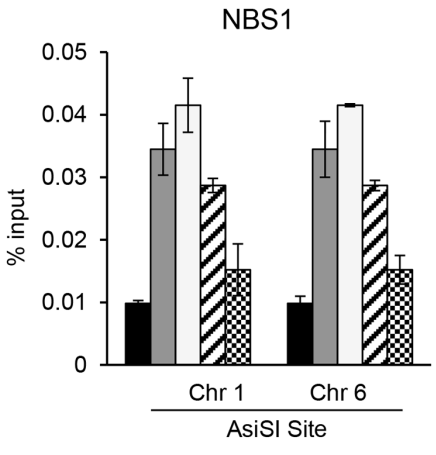

24h Rec
Q $48 \mathrm{~h} \operatorname{Rec} \quad$ \% $72 \mathrm{~h} R e c$

Figure 5: Cell-cycle re-entry induces a delayed resolution of DSBs. In panel A and B. cell cycle profiles and Ki67 levels detected by flow cytofluorimetry of $\mathrm{G}_{0}$ MCF10-AsiSI-ER released into fresh medium and collected as indicated. C. Cell cycle distribution of $\mathrm{G}_{0}-$ arrested MCF10A-AsiSI-ER cells treated for $2 \mathrm{~h}$ with 4OHT, then released into fresh medium and collected as indicated. DNA content of propridium iodide stained cells was determined by flow cytofluorimetry. D. Western blotting of MCF10A-AsiSI-ER cells treated as above. E. ChIP against $\gamma \mathrm{H} 2 \mathrm{AX}$ and NBS1 in MCF10A-AsiSI-ER treated for $2 \mathrm{~h}$ with 4OHT, then released into fresh medium, collected as indicated and analyzed by qPCR. Data are from independent experiments with $\mathrm{SD}(n=3)$. 
that $\gamma \mathrm{H} 2 \mathrm{AX}$ and NBS1 were rapidly recruited to the AsiSI sites and, most importantly, reduction of these DSBs factors followed a kinetics similar to that observed in asynchronously proliferating cells (compare Figure $6 \mathrm{~B}$ and Figure 2C). The data demonstrated that, unlike damaged $\mathrm{G}_{\mathrm{o}}$-arrested cells, $\mathrm{G}_{1}$-damaged cells exhibit efficient resolution of DSBs.

Because DSBs assembly of DDR repair factors occurs within minutes following the DNA damage event, we comparatively determined the timing of $\gamma \mathrm{H} 2 \mathrm{AX}$, NBS1 and XRCC4 recruitment in cells exposed to AsiSIdamage for a short period of time (20') in asynchronously proliferating, $\mathrm{G}_{0}-$, or $\mathrm{G}_{1}$-arrested cells. Figure 7 shows that early recruitment of these factors is largely similar in all the three cell populations analyzed. Thus, the initial recognition of DSBs and assembly of DSB factors is largely similar regardless of the cell cycle phase during which the DSB is produced.

A

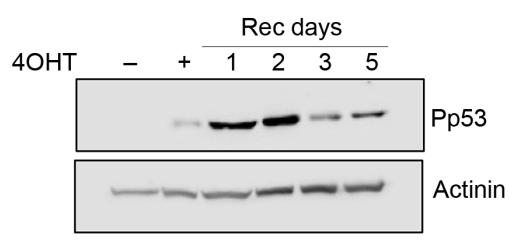

B

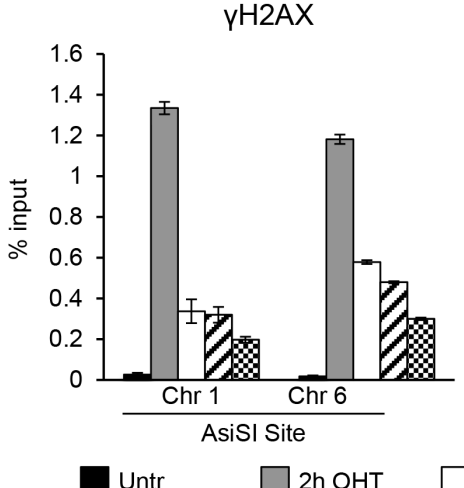

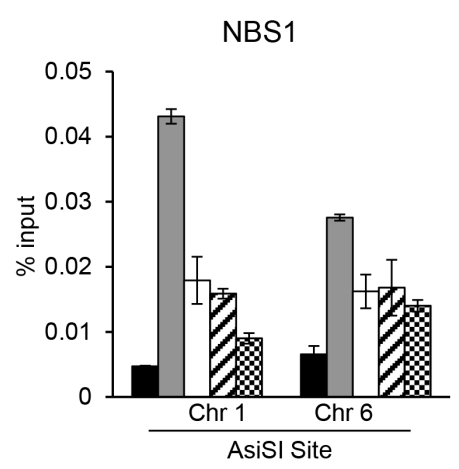

$\square 48 \mathrm{~h} \operatorname{Rec} \quad$ \& $72 \mathrm{~h} \operatorname{Rec}$

Figure 6: DSBs induced at G1/S phase. Synchronized cells were exposed for $2 \mathrm{hr}$ to $4 \mathrm{OHT}$ and then allowed to recovery for the indicted times. A. total cell extracts from G1/S phase MCF10A-AsiSI before and the indicated times after 4OHT removal were probed with anti-phospho-p53 and actinin as loading control. Panel B. ChIP against $\gamma \mathrm{H} 2 \mathrm{AX}$ and NBS1 in MCF10-AsiSI-ER analyzed by qPCR. Data are from independent experiments with $\mathrm{SD}(n=3)$.

$\gamma \mathrm{H} 2 \mathrm{AX}$

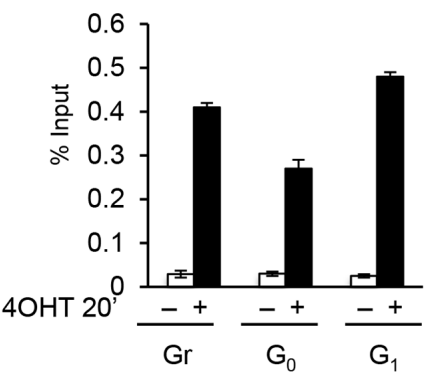

NBS1

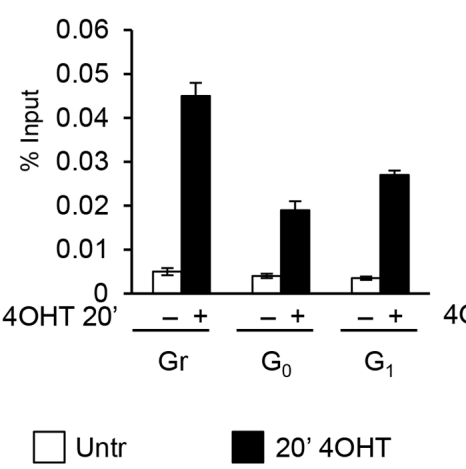

XRCC4

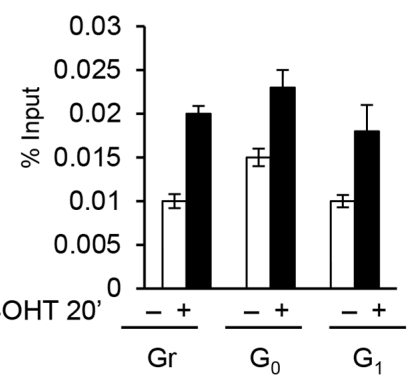

Figure 7: ChIP analysis with $\gamma \mathrm{H} 2 \mathrm{AX}$, NBS1 and XRCC4 antibodies in MFC10AsiSI-ER cells after a short pulse of 4OHT treatment $\left(\mathbf{2 0}^{\prime}\right)$. The values reported were calculated as percentage of input. Error bars indicate SD for three independent experiments. 


\section{DISCUSSION}

Here we report a comparative study of DSB response occurring at specific stages of the cell cycle. We generated a human normal non-tumorigenic epithelial MCF10A cell line expressing the estrogen receptorinducible AsiSI restriction enzyme, which allows the study of the wave of repair events occurring during specific stages of the cell cycle. We found that DSBs occurring in $\mathrm{G}_{0}$ cells are irreparable and $\mathrm{G}_{1} / \mathrm{S}$ transition is required for complete DNA damage resolution. We demonstrated that the $\mathrm{G}_{0}$ cells retain a functional DDR but, lacking DNA repair competence, they may accumulate DNA damage, which could reach critical levels and triggers the apoptotic cascade.

In agreement with previous studies in U2OS [22, 30-33], AsiSI dependent DSBs in proliferating MCF10A induce canonical DDR activation, which is followed by progressive resolution of DSBs. Conversely, we found that DSB induction in $\mathrm{G}_{0}$-arrested cells leads to efficient $\gamma \mathrm{H} 2 \mathrm{AX}$ and 53BP1 foci formation, but differently from asynchronous cells, damage is never repaired. Damaged $\mathrm{G}_{0}$-arrested cells show a robust and irreversible activation of the phospho-p53/p21 axis, and undergo apoptosis one week after DSBs induction. Through ChIP-sequencing we found that proliferating and $\mathrm{G}_{0}$ damaged cells showed the same number of DSB domains with similar enrichment of $\gamma \mathrm{H} 2 \mathrm{AX}$, demonstrating that the efficiency of DSBs induced in either proliferating or $\mathrm{G}_{0}$-arrested cells is largely similar. Moreover, ChIP experiments revealed that in $\mathrm{G}_{0}$ damaged cells the levels of $\gamma \mathrm{H} 2 \mathrm{AX}$, NBS1 and XRCC4 recruited to DSBs were largely similar to synchronous proliferating cells; thus, the cell cycle phase does not interfere with the initial steps of DNA damage response. However, the persistent accumulation of repair factors at DSBs indicates that DNA repair resolution is compromised in $\mathrm{G}_{0}$ cells. The inability of $\mathrm{G}_{\mathrm{o}}$ cells to repair damage was not due to altered expression of DDR genes, since $G_{0}$-arrested cells show similar expression levels of different repair factors when compared to proliferating cells, with the exception of RAD51 expression. Lack of RAD51 expression in $G_{0}$ cells is consistent with the notion that HR, which relays on RAD51 activity is efficient in S and $\mathrm{G}_{2}$ cell cycle phase, but limited in $\mathrm{G}_{0} / \mathrm{G}_{1}$ [14-16].

Most notably, we find that cell cycle re-entry of $\mathrm{G}_{0}$ damaged cells restored DNA repair competence, but led to delayed resolution of DSBs compared to proliferating cells, suggesting that $G_{0}$-damaged cells required $G_{1} / S$ transition to complete DSBs repair. Accordingly, resolution of DSBs induced in synchronized $\mathrm{G}_{1} / \mathrm{S}$ cells occurred with kinetic similar to that observed in proliferating cells.

Here we demonstrated that DDR activation does not depend on the phase of the cell cycle in which the DSB is generated. Similarly, a recent work reported that in IR-exposed fibroblasts, quiescence does not affect the DNA damage response, and activation of p53 and phosphorylation of $\gamma \mathrm{H} 2 \mathrm{AX}$ are similar between proliferating and quiescent cells [34].

Our data reveal that in $\mathrm{G}_{0}$ most of examined DDR factors are expressed at levels comparable to those observed in proliferating cells, and NHEJ is the main repair pathway since RAD51, a critical component of $\mathrm{HR}$, is undetectable in Go. However, the $\mathrm{G}_{1} / \mathrm{S}$ transition is required to complete resolution of DSBs induced in $\mathrm{G}_{0}$ cells. A possible explanation is that some DSBs induced in $G_{1}$ are repaired by HR as cells progress to $S$ phase [35]. Clearly, different molecular mechanisms involved in DNA repair occurring at specific cell cycle phases have been evolved, and the DDR differs in mitotic and interphase cells. It has been shown that DDR is dampened during mitosis. During mitosis DDR is inhibited to prevent telomere fusion and entry into mitosis in the presence of unrepaired DNA can lead to cell death, thus DDR clearly differs in mitotic and interphase cells [25-28]

Mammalian tissues and organs of consist of different cell types, including dividing, non-dividing and stem cells. Terminally differentiated cells are permanently withdrawn from the cell cycle and partly resistant to apoptosis [36, 37]. d'Adda di Fagagna and collaborators showed that terminally differentiated astrocytes exhibit radio-resistance and strongly attenuated expression of most of DDR genes compared to undifferentiated progenitors [38]. It has been shown that in IR-exposed quiescent myoblast the ATM-p53 axis operates normally, while it is compromised in differentiated myotubes [39], indicating that the lack of a robust DDR and radio-resistance can be linked to the terminal differentiation and irreversible exit from the cell cycle. We cannot exclude, however, that differences in DNA damage response in cultured $\mathrm{G}_{0}$-arrested cells and in terminally differentiated non-proliferating cells are strictly cell type-specific and depend on the physiological context.

Our findings also help to dispel the dogma that completion of DNA damage repair is the essential condition for entry in the next phase of the cell cycle and stresses the notion the cell cycle position of a damage cell affects the repair competence. Further investigations are needed to understand mechanisms that coordinate repair in proliferating, quiescent and terminally differentiated cells to preserve genome integrity.

\section{MATERIALS AND METHODS}

\section{Cell cultures and retroviral infection}

MCF10A were cultured in 1:1 mixture DMEM-F12 supplemented with 5\% horse serum, $10 \mu \mathrm{g} / \mathrm{ml}$ insulin, $0,5 \mu \mathrm{g} / \mathrm{ml}$ hydrocortisone, $100 \mathrm{ng} /$ $\mathrm{ml}$ cholera enterotoxin, and $20 \mathrm{ng} / \mathrm{ml}$ epidermal growth factor, and incubated at $37^{\circ} \mathrm{C}$ in humidified atmosphere with 5\% CO2. To generate MCF10Asi-ER cells the pBABE-HA-AsiSI-ER plasmid was transfected into 
$293 \mathrm{~T}$ cells expressing the structural components for retrovirus packaging, medium was harvested after $36 \mathrm{~h}$, filtered and used to infect MFC10 cells and selection performed using $1 \mu \mathrm{g} / \mathrm{ml}$ puromycin; single cell clones were isolated and analyzed.

\section{Cell cycle synchronization}

MCF10A-AsiSI cells were arrested in $\mathrm{G}_{0}$ by growth in minimal medium (1:1 mixture DMEM-F12 supplemented with $5 \%$ horse serum) for two days. To induce re-entry into cell cycle, the $\mathrm{G}_{\mathrm{O}}$ arrested cells were cultured in complete medium and cell cycle re-entry was monitored by flow cytometry analysis and Ki67 content.

\section{Antibodies}

The antibodies used for different applications in this study are listed in Supplementary Table S1.

\section{Flow cytometry analysis}

To analyze the DNA profile, cells were fixed in methanol at $-20^{\circ} \mathrm{C}$ and stained in hypotonic solution of $0,1 \%$ Na-Citrate, $50 \mu \mathrm{g} / \mathrm{ml}$ propidium iodide, $50 \mu \mathrm{g} / \mathrm{ml}$ RNAse and $0,00125 \%$ NP40 for 30 ' at room temperature. For Ki67 quantification cells were permealyzed with $0,1 \%$ Triton $\mathrm{X}-100 / \mathrm{PBS}$, blocked in 5\% Bovine Serum Albumin/PBS and stained with the primary antibody anti-Ki67; then, cells were incubated with the secondary antibody Alexa647 Donkey anti-goat (Invitrogen) before propidium iodide staining. Cytofluorimetric acquisition and analysis were performed on a Becton Dickinson FACScalibur flow cytometer using FACSDiva, CellQuest Pro and ModFit LT 3.0 software.

\section{Western blot analysis}

Whole-cell extracts were obtained using buffer $\mathrm{F}$ (10 $\mathrm{mM}$ TrisHCl pH 7.5, $150 \mathrm{mM} \mathrm{NaCl}, 30 \mathrm{mM} \mathrm{Na} 4 \mathrm{O} 7 \mathrm{P} 2$, $50 \mathrm{mM}$ NaF, $5 \mathrm{mM} \mathrm{ZnCl} 2,0.1 \mathrm{mM} \mathrm{Na} 3 \mathrm{VO} 4,1 \%$ Triton, $0.1 \mathrm{mM}$ PMSF). $50 \mu \mathrm{g}$ of protein extracts were loaded and separated by SDS-PAGE and WB was performed with indicated antibodies.

\section{Immunofluorescence}

Immunofluorescences of MFC10AsiSI-ER cells were performed as previously described. Briefly, cells were fixed in $4 \%$ paraformaldehyde in PBS, permeabilized in $0.1 \%$ Triton X-100 in PBS, pre-blocked in 2\% BSA$3 \% \mathrm{NS}-\mathrm{PBS}$ for $30 \mathrm{~min}$ at room temperature, and then incubated for $1 \mathrm{~h}$ at $37^{\circ} \mathrm{C}$ with mouse anti-HA and rabbit anti- $\gamma \mathrm{H} 2 \mathrm{AX}$ for $30^{\prime}$ at $37^{\circ} \mathrm{C}$ anti-53BP 1 . Primary antibodies were detected by incubation with $\mathrm{Cy} 3-$ coniugated anti-mouse or FITC-conjugated anti-rabbit antibody. Images were acquired using a Nikon Eclipse TE 2000-U microscope.

\section{Chromatin immunoprecipitation assays}

ChIP experiments with chromatin extracts from MCF10-AsiSIER cells were performed as described [32]. IPs materials were analyzed in duplicate by quantitative PCR, using Syber Green 2X PCR Master Mix (Applied Biosystem). For qPCRs $3 \mu \mathrm{l}$ out of $150 \mu \mathrm{l}$ immunoprecipitated DNA was used. The antibodies are listed in Table S1. After reversal of the crosslinks, the immunoprecipitated DNA was quantified by qPCR with the primer sets described in Table S2. For each ChIP assay a control amplicon from Chromosome X (Table S2) was used.

\section{Chip-sequencing, mapping and peak analysis}

ChIP-seq libraries were prepared from $10 \mathrm{ng}$ of ChIP (or Input) DNA with TruSeq ChIP Sample Prep Kit (Illumina) according to the manufacturer's instructions. Prior to sequencing, libraries were quantified using Quibit (Invitrogen) and quality-controlled using Agilent's Bioanalyzer. 50bp single-end sequencing was performed using Illumina HiSeq 2000 platform (Genomix4life S.R.L., Baronissi, Salerno, Italy) according to standard operating procedures. Alignments were performed with BWA [40] to hg18 using default parameters. SAMtools [41] and BEDtools [42] were used for filtering steps and file formats conversion. The peaks were identified from uniquely mapped reads without duplicates using MACS and the p-value cutoff used for peak detection was 1e-5. DNA Input was used as control. UCSC genome browser was used for data visualization. To plot data of average profiles around DSBs, AsiSI site positions were retrieved from the human genome (hg18). ChIP-seq counts were retrieved for $10 \mathrm{~kb}$ around each of these DSBs and averaged with a 200-bp window using a custom R-script [43]. ChIPseq data were deposited to NCBI GEO and are available under accession number GSE71447

\section{RNA extraction and qRT-PCR quantification}

RNA was extracted from MCF10A-AsiSIER cells using EuroGold Trifast (EuroClone). cDNA was generated using Quantitec Reverse Transcription Kit (Qiagen), according to manufacturer's protocol. Quantitative analysis was performed using SYBR Green 2X PCR Master Mix (Applied Biosystem). Each sample was run in triplicate and normalized to the expression of housekeeping beta-glucoronidase (GUS) gene as previously described [44]. Primers are presented in Supplementary Table S2.

\section{ACKNOWLEDGMENTS}

We are grateful to Gaelle Legube from providing the HA-AsiSI-ER vector. This work was supported by grants 
AIRC (IG 13173) to B.M., from Epigenomics Flagship Project-EPIGEN, C.N.R. and P.O.R. Campania FSE 2007-2013 Project MoVIE to L.L.

\section{Editorial note}

This paper has been accepted based in part on peerreview conducted by another journal and the authors' response and revisions as well as expedited peer-review in Oncotarget.

\section{CONFLICTS OF INTEREST}

The authors declare no conflicts of interest.

\section{REFERENCES}

1. Berkovich E, Monnat RJ, Jr., Kastan MB. Roles of ATM and NBS1 in chromatin structure modulation and DNA double-strand break repair. Nature Cell Biology. 2007; 9:683-690.

2. Matsuoka S, Ballif BA, Smogorzewska A, McDonald ER, 3rd, Hurov KE, Luo J, Bakalarski CE, Zhao Z, Solimini N, Lerenthal Y, Shiloh Y, Gygi SP, Elledge SJ. ATM and ATR substrate analysis reveals extensive protein networks responsive to DNA damage. Science. 2007; 316:1160-1166.

3. Thompson LH. Recognition, signaling, and repair of DNA double-strand breaks produced by ionizing radiation in mammalian cells: the molecular choreography. Mutation Research. 2012; 751:158-246.

4. Price BD, D'Andrea AD. Chromatin remodeling at DNA double-strand breaks. Cell. 2013; 152:1344-1354.

5. Rogakou EP, Pilch DR, Orr AH, Ivanova VS, Bonner WM. DNA double-stranded breaks induce histone H2AX phosphorylation on serine 139. The Journal of Biological Chemistry. 1998; 273:5858-5868.

6. Gospodinov A, Herceg Z. Chromatin structure in double strand break repair. DNA Repair. 2013; 12:800-810.

7. Gospodinov A, Herceg Z. Shaping chromatin for repair. Mutation Research. 2013; 752:45-60.

8. Fradet-Turcotte A, Canny MD, Escribano-Diaz C, Orthwein A, Leung CCY, Huang H, Landry M-C, Kitevski-LeBlanc J, Noordermeer SM, Sicheri F, Durocher D. 53BP1 is a reader of the DNA-damage-induced H2A Lys 15 ubiquitin mark. Nature. 2013; 499:50-54.

9. Bartek J, Lukas J. DNA damage checkpoints: from initiation to recovery or adaptation. Curr. Opin. Cell Biol. 2007; 19:238-245

10. Jackson SP, Bartek J. The DNA-damage response in human biology and disease. Nature. 2009; 461:1071-1078.

11. Li L, Clevers H. Coexistance of quiescent and active adult stem cells in mammals. Science. 2010; 327:542-545.
12. Cheung TH, Rando TA. Molecular regulation of stem cell quiescence. Nat. Rev. Mol. Cell. Biol. 2013; 14:329-340.

13. Coller HA, Sang L, Roberts JM. a new description of cellular quiescence. PloS Biol. 2006; 4:e83

14. Iyama $\mathrm{T}$, Wilson DM, 3rd. DNA repair mechanisms in dividing and non-dividing cells. DNA Repair. 2013; 12:620-636.

15. Aparicio T, Baer R, Gautier J. DNA double-strand break repair pathway choice and cancer. DNA Repair. 2014; 19:169-175.

16. Branzei D, Folani M. Regulation of DNA repair throughout the cell cycle. Nat. Rev. Mol. Cell. Biol. 2008; 9:297-308.

17. Escribano-Diaz C, Durocher D. DNA repair pathway choice - a PTIP of the hat to 53BP1. EMBO Rep. 2013; 14:665-666.

18. Escribano-Diaz C, Orthwein A, Fradet-Turcotte A, Xing M, Young JTF, Tkac J, Cook MA, Rosebrock AP, Munro M, Canny MD, Xu D, Durocher D. A cell cycle-dependent regulatory circuit composed of 53BP1-RIF1 and BRCA1CtIP controls DNA repair pathway choice. Molecular cell. 2013; 49:872-883.

19. Radhakrishnan SK, Jette N, Lees-Miller SP. Nonhomologous end joining: emerging themes and unanswered questions. DNA Repair. 2014; 17:2-8.

20. Shibata A, Moiani D, Arvai AS, Perry J, Harding SM, Genois MM, Maity R, van Rossum-Fikkert S, Kertokalio A, Romoli F, Ismail A, Ismalaj E, Petricci E, et al. DNA double-strand break repair pathway choice is directed by distinct MRE11 nuclease activities. Molecular Cell. 2014; 53:7-18.

21. Panier S, Durocher D. Push back to respond better: regulatory inhibition of the DNA double-strand break response. Nat Rev Mol Cell Biol. 2013; 14:661-672.

22. Aymard F, Bugler B, Schmidt CK, Guillou E, Caron P, Briois S, Iacovoni JS, Daburon V, Miller KM, Jackson SP, Legube G. Transcriptionally active chromatin recruits homologous recombination at DNA double-strand breaks. Nature Structural \& Molecular Biology. 2014; 21:366-374.

23. Miller KM, Jackson SP. Histone marks: repairing DNA breaks within the context of chromatin. Biochemical Society transactions. 2012; 40:370-376.

24. Soria G, Polo SE, Almouzni G. Prime, repair, restore: the active role of chromatin in the DNA damage response. Molecular Cell. 2012; 46:722-734.

25. Terasawa M, Shinohara A, Shinohara M. Canonical nonhomologopus end joining in mitosis induces genome instability and is suppressed by M-phase-specific phopshorylation of XRCC4. Plos Genetics. 2014; 10e104563.

26. Orthwein A, Fradet-Turcotte A, Noordermeer SM, Canny MD, Brun CM, Strecker J, Escribano-Diaz C, Durocher D. Mitosis Inhibits DNA Double-Strand Break Repair to Guard Against Telomere Fusions. Science. 2014; 344:189-193. 
27. Lees-Miller SP. DNA double strand break repair in mitosis is suppressed by phosphorylation of XRCC4. PLoS Genetics. 2014; 10:e1004598.

28. Cesare AJ. Mitosis, double strand break repair, and telomeres: a view from the end: how telomeres and the DNA damage response cooperate during mitosis to maintain genome stability. BioEssays. 2014; 36:1054-1061.

29. Caron P, Aymard F, Iacovoni JS, Briois S, Canitrot Y, Bugler B, Massip L, Losada A, Legube G. Cohesin protects genes against gammaH2AX Induced by DNA double-strand breaks. PLoS Genetics. 2012; e1002460.

30. Iacovoni JS, Caron P, Lassadi I, Nicolas E, Massip L, Trouche D, Legube G. High-resolution profiling of gammaH2AX around DNA double strand breaks in the mammalian genome. The EMBO Journal. 2010; 29:1446-1457.

31. Massip L, Caron P, Iacovoni JS, Trouche D, Legube G. Deciphering the chromatin landscape induced around DNA double strand breaks. Cell cycle. 2010; 9:2963-2972.

32. Ambrosio S, Amente S, Napolitano G, Di Palo G, Lania L, Majello B. MYC impairs resolution of site-specific DNA double-strand breaks repair. Mutation research. 2015; 774:6-13.

33. Napolitano G, Amente S, Lavadera ML, Di Palo G, Ambrosio S, Lania L, Dellino GI, Pelicci PG, Majello B. Sequence-specific double strand breaks trigger P-TEFbdependent Rpb1-CTD hyperphosphorylation. Mutation Research. 2013; 749:21-27.

34. Dai J, Itahana K, Baskar R. Quiescence does not affect p53 and stress response by irradiation in human lung fibroblasts. Biochemical and Biophysical Research Communications. 2015; 458:104-109.

35. Karanam K, Kafri R, Loewer A, Lahav G. Quantitative live cell imaging reveals a gradual shift between DNA repair mechanisms and a maximal use of HR in mid-S phase. Mol. Cell. 2012; 47: 320-329.

36. Buttitta LA, Edgar BA. Mechanisms controlling cell cycle exit upon terminal differentiation. Curr Opin Cell Biol. 2007; 19: 697-704.

37. Fortini P, Dogliotti E. Mechanisms of dealing with DNA damage in terminally differentiated cells. Mutation Research. 2010; 685:38-44.

38. Schneider L, Fumagalli M, d'Adda di Fagagna F. Terminally differentiated astrocytes lack DNA damage response signaling and are radioresistant but retain DNA repair proficiency. Cell Death and Differentiation. 2012; 19:582-591.

39. Latella L, Lukas J, Simone C, Puri PL, Bartek J. Differentiation-Induced Radioresistance in Muscle Cells. Molecular and Cellular Biology. 2004; 24:6350-636.

40. Li H, Durbin R. Fast and accurate short read alignment with Burrows-Wheeler transform. Bioinformatics. 2009; 25:1754-1760.

41. Li H, Handsaker B, Wysoker A, Fennell T, Ruan J, Homer N, Marth G, Abecasis G, Durbin R, Genome Project Data Processing S. The Sequence Alignment/Map format and SAMtools. Bioinformatics. 2009; 25:2078-2079.

42. Quinlan AR, Hall IM. BEDTools: a flexible suite of utilities for comparing genomic features. Bioinformatics. 2010; 26:841-842.

43. Scala G, Affinito O, Miele G, Monticelli A, Cocozza S. Evidence for evolutionary and nonevolutionary forces shaping the distribution of human genetic variants near transcription start sites. PloS one. 2014; 9:e114432.

44. Amente S, Bertoni A, Morano A, Lania L, Avvedimento EV, Majello B. LSD1-mediated demethylation of histone H3 lysine 4 triggers Myc-induced transcription. Oncogene. 2010; 29:3691-3702. 